\title{
AN INTEGRAL INVOLVING THE PRODUCT OF A BESSEL FUNCTION AND AN E-FUNCTION
}

\author{
$b y$ FOUAD M. RAGAB \\ (Received 22nd June, 1950)
}

The formula *

$$
\begin{aligned}
& 4 \int_{0}^{\infty} \lambda^{m-1} K_{n}(2 \lambda) E\left(p ; \alpha_{r}: q ; \rho_{s}: x \lambda^{-2}\right) d \lambda \\
& =E\left(p+2 ; \alpha_{r}: q ; \rho_{s}: x\right),
\end{aligned}
$$

where $\alpha_{p+1}=\frac{1}{2}(m+n), \alpha_{p+2}=\frac{1}{2}(m-n), R(m \pm n)>0$ and $x$ is real and positive, was given by MacRobert (Phil. Mag., Ser. 7, XXXI, p. 258). From it the formula (6) below will be deduced.

In (1) let it be assumed that $R(m \pm n)>0, R\left(\frac{3}{2}-m+2 \alpha_{r}\right)>0, r=1,2, \ldots, p$, and let $\operatorname{amp} \lambda$ decrease by $\frac{1}{2} \pi$, amp $x$ decreasing simultaneously by $\pi$, finally writing $\lambda / i$ in place of $\lambda$ and $x e^{-i \pi}$ in place of $x:$ then, noting that

we have

$$
K_{n}(t)=i^{n} G_{n}(i t)
$$

$$
\begin{aligned}
& 4 i^{n-m} \int_{0}^{\infty} \lambda^{m-1} G_{n}(2 \lambda) E\left(p ; \alpha_{r}: q ; \rho_{s}: x \lambda^{-2}\right) d \lambda \\
& =E\left(p+2 ; \alpha_{r}: q ; \rho_{s}: x e^{-i \pi}\right) .
\end{aligned}
$$

Similarly, on increasing amp $\lambda$ by $\frac{1}{2} \pi$ and amp $x$ by $\pi$, we have

$$
\begin{aligned}
& 4 i^{n+m} \int_{0}^{\infty} \lambda^{m-1} G_{n}\left(2 \lambda e^{i \pi}\right) E\left(p ; \alpha_{r}: q ; \rho_{s}: x \lambda^{-2}\right) d \lambda \\
& =E\left(p+2 ; \alpha_{r}: q ; \rho_{s}: x e^{i \pi}\right)
\end{aligned}
$$

Hence, on applying the formula

it is found that

$$
\pi i J_{n}(t)=G_{n}(t)-i^{2 n} G_{n}\left(t e^{i \pi}\right)
$$

$$
\begin{aligned}
4 i \pi \int_{0}^{\infty} \lambda^{m-1} J_{n}(2 \lambda) E\left(p ; \alpha_{r}: q: \rho_{s}: x \lambda^{-2}\right) d \lambda \\
\quad=i^{m-n} E\left(p+2 ; \alpha_{r}: q ; \rho_{s}: x e^{-i \pi}\right) \\
-i^{-m+n} E\left(p+2 ; \alpha_{r}: q ; \rho_{s}: x e^{i \pi}\right),
\end{aligned}
$$

where $R(m+n)>0, R\left(\frac{3}{2}-m+2 \alpha_{r}\right)>0, r=1,2, \ldots, p$, and $x$ is real and positive.

In particular, if $p \geqq q-1$, formula (6) can be written

$$
\begin{aligned}
& 2 \pi \int_{0}^{\infty} \lambda^{m-1} J_{n}(2 \lambda) E\left(p ; \alpha_{r}: q ; \rho_{s}: x \lambda^{-2}\right) d \lambda \\
& =\sum_{r=1}^{p+1} \frac{\prod_{s=1}^{p+2} \Gamma\left(\alpha_{s}-\alpha_{r}\right)}{\prod_{t=1}^{q} \Gamma\left(\rho_{t}-\alpha_{r}\right)} \Gamma\left(\alpha_{r}\right) \sin \left(\frac{1}{2} m-\frac{1}{2} n-\alpha_{r}\right) \pi x^{\alpha_{r}} \\
& \times F\left\{\begin{array}{l}
\alpha_{r}, \alpha_{r}-\rho_{1}+1, \ldots, \alpha_{r}-\rho_{q}+1 ;(-1)^{p-q+1} x \\
\alpha_{r}-\alpha_{1}+1, \ldots * \ldots, \alpha_{r}-\alpha_{p+2}+1
\end{array}\right\}
\end{aligned}
$$

* For the properties of the E-functions see MacRobert, Functions of a Complex Variable, third edition. 
where $R(m+n)>0, R\left(\frac{3}{2}-m+2 \alpha_{r}\right)>0, r=1,2, \ldots, p$, and $x$ is real and positive. It should be noted that the $(p+2)$ th term on the right of $(7)$ does not appear because $\alpha_{p+2}=\frac{1}{2}(m-n)$.

If $m=\beta+1, n=\beta-1$ and $\rho_{a}=\beta$, then

and

$$
\begin{gathered}
\alpha_{p+1}=\beta, \alpha_{p+2}=1 \\
\alpha_{r}-\alpha_{p+1}+1=\alpha_{r}-\beta+1,
\end{gathered}
$$

which cancels $\alpha_{r}-\rho_{q}+1$ on the right of (7).

Also

$$
\alpha_{r}-\alpha_{p+2}+1=\alpha_{r}
$$

which cancels $\alpha_{r}$ on the right of (7).

Again, $\rho_{q}-\alpha_{p+1}=0$, so that

$$
\frac{1}{\Gamma\left(\rho_{q}-\alpha_{p+1}\right)}=0,
$$

and therefore the last term on the right of (7) disappears.

Finally, noting that

$$
\Gamma\left(\alpha_{p+2}-\alpha_{r}\right) \Gamma\left(\alpha_{r}\right)=\frac{\pi}{\sin \left(\alpha_{r} \pi\right)},
$$

that

and that

$$
\sin \left(\frac{1}{2} m-\frac{1}{2} n-\alpha_{r}\right) \pi=\sin \left(\alpha_{r} \pi\right),
$$

we have, if $p \geqq q-1$,

$$
\frac{\Gamma\left(\alpha_{p+1}-\alpha_{r}\right)}{\Gamma\left(\rho_{q}-\alpha_{r}\right)}=1
$$

$$
\begin{aligned}
& 2 \int_{0}^{\infty} \lambda^{\beta} J_{\beta-1}(2 \lambda) E\left(p ; \alpha_{r}: q ; \rho_{s}: x \lambda^{-2}\right) d \lambda \\
& =\sum_{r=1}^{p} \frac{\prod_{g=1}^{p} \Gamma\left(\alpha_{s}-\alpha_{r}\right)}{\prod_{t=1}^{q-1} \Gamma\left(\rho_{t}-\alpha_{r}\right)} x^{\alpha_{r}} F\left(\begin{array}{l}
\alpha_{r}-\rho_{1}+1, \ldots, \alpha_{r}-\rho_{q-1}+1 ;(-1)^{p-q+1} x \\
\alpha_{r}-\alpha_{1}+1, \ldots * \ldots, \alpha_{r}-\alpha_{p}+1
\end{array}\right),
\end{aligned}
$$

where $\rho_{q}=\beta, R(\beta)>0, R\left(\frac{1}{2}-\beta+2 \alpha_{r}\right)>0, r=1,2, \ldots, p$.

It should be noted that $\beta$ does not appear on the right of (8).

This result was given, for the case $p=q+1$, by Meijer (Proc. Akad. te Amsterdam, XXXIX, 1936, p. 397).

UNIVERSITY OF GLASGOW 